\title{
Pd/C-catalyzed, copper-free Sonogashira coupling: one-pot synthesis of 1-aryl-4-(2-phenylethynyl)[1,2,4]triazolo[4,3-a]- quinoxalines in water
}

\author{
Mohammad Bakherad • Saeideh Jajarmi
}

Received: 1 June 2012/Accepted: 14 December 2012/Published online: 14 February 2013

(C) The Author(s) 2013. This article is published with open access at Springerlink.com

\begin{abstract}
Copper-free, $\mathrm{Pd} / \mathrm{C}$-catalyzed, one-pot reaction of 2,3-dichloroquinoxaline with hydrazine hydrate, bromine, phenylacetylene, and a variety of aldehydes provides an efficient and direct method for the preparation of 1-aryl4-(2-phenylethynyl)[1,2,4]triazolo[4,3-a]quinoxalines in water at $70{ }^{\circ} \mathrm{C}$. This methodology involves the use of inexpensive reagents or catalysts, and thus permits a new and practical access to triazolo[4,3-a]quinoxalines.
\end{abstract}

Keywords Palladium catalyst - Triazoloquinoxaline . Sonogashira reaction - Phenylacetylene

\section{Introduction}

Quinoxaline and its derivatives show a broad spectrum of biological activities including anti-tumor [1], anti-viral [2], anti-tuberculosis [3], and anti-inflammatory [4] activities, and, hence, are an important class of nitrogen-containing heterocycles, and useful intermediates in organic synthesis [5]. Furthermore, triazolo[4,3-a]quinoxalines have been shown to be potent and selective adenosine receptor ligands [6].

The Sonogashira reaction catalyzed by palladium and copper is a powerful and straightforward method for the construction of arylated internal alkyne compounds [7-9]. These are important intermediates in organic synthesis and for the preparation of natural products [10], biologically active molecules [11], molecular electronics [12], and polymers [13].

M. Bakherad $(\bowtie) \cdot$ S. Jajarmi

School of Chemistry, Shahrood University of Technology,

Shahrood, Iran

e-mail:m.bakherad@yahoo.com
Typically, these coupling-cyclization reactions are carried out using a palladium catalyst [e.g., $\mathrm{Pd}\left(\mathrm{PPh}_{3}\right)_{4}$, $\left(\mathrm{PPh}_{3}\right)_{2} \mathrm{PdCl}_{2}$, etc.] and a copper salt as the co-catalyst in the presence of an amine base. The use of palladium on charcoal as a catalytic system for the efficient Sonogashira coupling of aryl halides with terminal alkynes has also been reported [14-17]. Compared to the frequently used expensive palladium catalysts [e.g., $\mathrm{Pd}\left(\mathrm{PPh}_{3}\right)_{4},\left(\mathrm{PPh}_{3}\right)_{2} \mathrm{PdCl}_{2}$, etc.], $\mathrm{Pd} / \mathrm{C}$-based methods have an economic advantage, and, hence, remain attractive for large or industrial scale preparations.

In recent years, a variety of modifications have been reported for this reaction, and significant progress has been made [18-22]. The most important modification is the elimination of the copper salt [23-27] since it can induce Glaser-type homocoupling of terminal alkynes in the presence of oxidants or air [28-30]. One of the powerful tools used to combine economic aspects with the environmental concerns is performing organic reactions in water; this strategy consists of two or more synthetic steps, which are carried out in water as a cheap, nontoxic, and environmentally friendly solvent, in a one-step reaction, without isolation of any intermediate, thus reducing time, and saving money, energy, and raw materials [31-33]. Several examples of $\mathrm{Pd}$-catalyzed Sonogashira reactions in aqueous media have been reported [34-37].

Recently, our group has prepared pyrrolo[2,3-b]quinoxaline derivatives under mild conditions in one-pot reactions catalyzed by $\mathrm{Pd}-\mathrm{Cu}$ [38-40]. We attempted to make this overall approach more attractive synthetically by examining the preparation of triazolo[4,3-a]quinoxalines using a one-pot reaction of 2,3-dichloroquinoxaline, hydrazine, aldehydes, bromine, and phenylacetylene catalyzed by $\mathrm{Pd} / \mathrm{C}$ without the use of copper as a co-catalyst in water. 


\section{Results and discussion}

We reported the synthesis of new derivatives of triazolo[4,3a]quinoxalines via $10 \%$ palladium/charcoal activated (Merck catalyst) catalyzed one-pot reaction of 2,3-dichloroquinoxaline, hydrazine, a suitable aldehyde, bromine, and phenylacetylene at $70{ }^{\circ} \mathrm{C}$ by employing water as the reaction medium. The use of water as a reaction medium represents remarkable benefits since this green solvent is highly polar and, therefore, immiscible with most organic compounds; moreover, the water-soluble by-products reside, and separation of the organic materials is thus easy.

Retrosynthetic analysis indicated that the desired 1-aryl4-(2-phenylethynyl) $[1,2,4]$ triazolo[4,3- $a]$ quinoxalines can also be synthesized from 2,3-dichloroquinoxaline, hydrazine, a suitable aldehyde, and phenylacetylene as the starting materials (Scheme 1).

For optimization of the reaction conditions, we studied the effect of various reaction parameters including the base, palladium catalyst, and temperature on the outcome of the copper-free reaction of 2,3-dichloroquinoxaline (1 equiv.) with hydrazine hydrate ( 2 equiv.), benzaldehyde (1 equiv.), bromine (1 equiv.), and phenylacetylene (1 equiv.) in the presence of $10 \% \mathrm{Pd} / \mathrm{C}(5 \mathrm{~mol} \%)$ and $\mathrm{K}_{2} \mathrm{CO}_{3}(2$ equiv.) in water at $70{ }^{\circ} \mathrm{C}$ (Table 1).

The reaction was influenced significantly by the base employed. It worked very well when inorganic bases such as $\mathrm{K}_{2} \mathrm{CO}_{3}$ and $\mathrm{KOH}$ were used (entries 2 and 3), with the best result obtained in the case of potassium carbonate. Two of the organic bases used, triethylamine and piperidine, also gave good yields (entries 5 and 8). It was found that while $\mathrm{PdCl}_{2}\left(\mathrm{PPh}_{3}\right)_{2}, \mathrm{PdCl}_{2} / \mathrm{PPh}_{3}, \mathrm{PdCl}_{2}$, and $\mathrm{Pd}_{2}(\mathrm{dba})_{3} \cdot \mathrm{CHCl}_{3}$ all catalyzed the reaction without the aid of any surfactant (entries 9-12), $10 \% \mathrm{Pd} / \mathrm{C}$ turned out to be the best catalyst in terms of yields (entry 2). When the catalyst loading was decreased to $3 \mathrm{~mol} \%$, the yield of $4 \mathbf{a}$ was $55 \%$ (entry 13). Increasing the amount of palladium catalyst did not increase the yield (entry 14). Decreasing the temperature to $25^{\circ} \mathrm{C}$ caused the yield to decrease to $35 \%$ (entry 15 ).

In order to examine the scope of this reaction, compound 1 was reacted with hydrazine, various aldehydes $\mathbf{2}$, bromine, and phenylacetylene in water at $70{ }^{\circ} \mathrm{C}$ to triazolo[4,3-a]quinoxalines in moderate to good yields (Table 2). When the less reactive acetylene, 1-hexyne, 1-octyne, and propargyl alcohol were used, Sonogashira coupling could not be achieved. The reactions were carried out under an argon atmosphere, and water was degassed prior to use.

Mechanistically, the formation of triazolo[4,3-a]quinoxalines 4 involves the following steps (Scheme 2): (i) formation of 1-aryl-4-chloro[1,2,4]triazolo[4,3-a]quinoxalines from the cyclization of intermediate $\mathbf{A}$ catalyzed by bromine [41]; and (ii) Sonogashira coupling reaction of $\mathbf{3}$ with phenyl acetylene.

\section{Conclusion}

In conclusion, the chemistry outlined here provides a simple, efficient, clean, and straightforward method for the synthesis of triazolo[4,3-a]quinoxaline derivatives via the copper-free, $\mathrm{Pd} / \mathrm{C}$-catalyzed reaction of readily available dichloroquinoxaline with hydrazine, bromine, phenylacetylene, and a variety of aldehydes in water. This methodology does not involve the use of expensive reagents or catalysts, and thus permits a new and practical access to triazolo[4,3- $a$ ]quinoxalines.

\section{Experimental}

All the compounds (phenylacetylene, aldehydes, $\mathrm{Pd} / \mathrm{C}$ ) were obtained from Merck. The reagents used were purchased from commercial suppliers, and used without further purification. Merck silica gel 60 was used for chromatography (230-400 mesh). The IR spectra were obtained as $\mathrm{KBr}$ pellets in the range of $400-4,000 \mathrm{~cm}^{-1}$ on a Shimadzu Model 460 spectrophotometer. ${ }^{1} \mathrm{H}$ NMR spectra were recorded at $400 \mathrm{MHz}$, and ${ }^{13} \mathrm{C}$ NMR spectra were recorded at $100 \mathrm{MHz}$ in DMSO- $d_{6}$. The chemical shifts are reported in $\operatorname{ppm}(\delta)$, and referenced to the residual proton signal for DMSO- $d_{6}(2.49 \mathrm{ppm})$. The coupling constants $(J)$ are reported in Hz. Mass spectrometric data (MS) were

Scheme 1

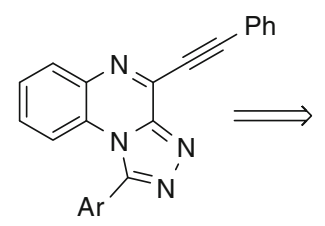<smiles>[C+]#CPc1ccccc1</smiles><smiles>Clc1nc2ccccc2n2c(Br)nnc12</smiles><smiles>C=CCCC</smiles><smiles>C=CC=NNc1nc2ccccc2nc1Cl</smiles><smiles>C=CC(Cl)=Nc1ccccc1Cl</smiles><smiles>[NH3+]Nc1nc2ccccc2nc1Cl</smiles> 
Table 1 Effect of base and catalyst on the heterocyclization during the reaction of 2,3-dichloroquinoxaline (1) with hydrazine hydrate, bromine, benzaldehyde, and phenylacetylene ${ }^{\mathrm{a}}$

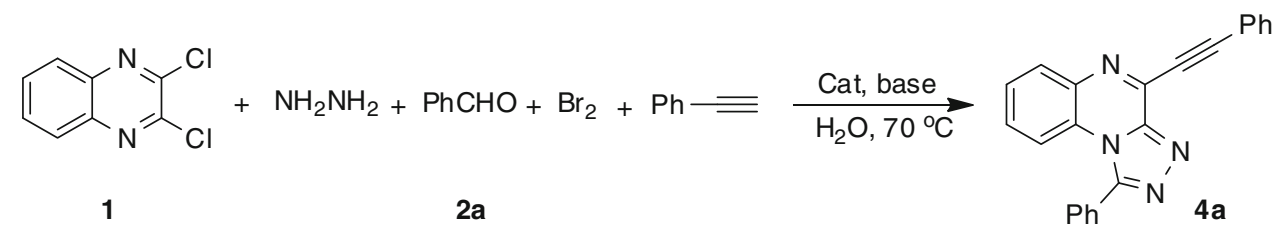

\begin{tabular}{|c|c|c|c|}
\hline Entry & Catalyst $/ \mathrm{mol} \%$ & Base & Yield $/ \%^{\mathrm{b}}$ \\
\hline 1 & $10 \% \mathrm{Pd} / \mathrm{C}(5)$ & $\mathrm{Cs}_{2} \mathrm{CO}_{3}$ & 75 \\
\hline 2 & $10 \% \mathrm{Pd} / \mathrm{C}(5)$ & $\mathrm{K}_{2} \mathrm{CO}_{3}$ & 82 \\
\hline 3 & $10 \% \mathrm{Pd} / \mathrm{C}(5)$ & $\mathrm{KOH}$ & 80 \\
\hline 4 & $10 \% \mathrm{Pd} / \mathrm{C}(5)$ & $\mathrm{Na}_{2} \mathrm{CO}_{3}$ & 78 \\
\hline 5 & $10 \% \mathrm{Pd} / \mathrm{C}(5)$ & $\mathrm{Et}_{3} \mathrm{~N}$ & 58 \\
\hline 6 & $10 \% \mathrm{Pd} / \mathrm{C}(5)$ & DIEA $^{c}$ & 42 \\
\hline 7 & $10 \% \mathrm{Pd} / \mathrm{C}(5)$ & Pyridine & 54 \\
\hline 8 & $10 \% \mathrm{Pd} / \mathrm{C}(5)$ & Piperidine & 60 \\
\hline 9 & $\mathrm{PdCl}_{2}(5) / \mathrm{PPh}_{3}(10)$ & $\mathrm{K}_{2} \mathrm{CO}_{3}$ & 50 \\
\hline 10 & $\mathrm{PdCl}_{2}\left(\mathrm{PPh}_{3}\right)_{2}$ & $\mathrm{~K}_{2} \mathrm{CO}_{3}$ & 70 \\
\hline 11 & $\operatorname{Pd}(\mathrm{dba})_{2}(5)$ & $\mathrm{K}_{2} \mathrm{CO}_{3}$ & 74 \\
\hline 12 & $\mathrm{PdCl}_{2}(5)$ & $\mathrm{K}_{2} \mathrm{CO}_{3}$ & 45 \\
\hline 13 & $10 \% \mathrm{Pd} / \mathrm{C}(3)$ & $\mathrm{K}_{2} \mathrm{CO}_{3}$ & 55 \\
\hline 14 & $10 \% \mathrm{Pd} / \mathrm{C}(10)$ & $\mathrm{K}_{2} \mathrm{CO}_{3}$ & 82 \\
\hline $15^{\mathrm{d}}$ & $10 \% \mathrm{Pd} / \mathrm{C}(5)$ & $\mathrm{K}_{2} \mathrm{CO}_{3}$ & 35 \\
\hline
\end{tabular}

a Reaction conditions: 1 (1.0 mmol), hydrazine hydrate $(2.0 \mathrm{mmol}), \mathbf{2 a}(1.0 \mathrm{mmol})$, bromine $(1.0 \mathrm{mmol})$, phenylacetylene $(1.0 \mathrm{mmol}), \mathrm{K}_{2} \mathrm{CO}_{3}$ (2.0 mmol), $70{ }^{\circ} \mathrm{C}, 12 \mathrm{~h}$

b Isolated yields

c Diisopropylethylamine

${ }^{\mathrm{d}}$ Reaction at $25^{\circ} \mathrm{C}$

obtained by electron ionization (EI, $70 \mathrm{eV}$ ) or electrospray ionization (ESI).

Typical procedure for the synthesis of 1-aryl-4(2-phenylethynyl) [1,2,4]triazolo[4,3-a]quinoxalines $4 a-4 k$

A mixture of $199 \mathrm{mg}$ 2,3-dichloroquinoxaline (1.0 mmol), $0.1 \mathrm{~cm}^{3}$ hydrazine hydrate $(99 \%, 2.0 \mathrm{mmol})$, a suitable aldehyde $(1.0 \mathrm{mmol})$, and $0.05 \mathrm{~cm}^{3}$ bromine $(1.0 \mathrm{mmol})$ were stirred in $5 \mathrm{~cm}^{3} \mathrm{H}_{2} \mathrm{O}$ at room temperature under an argon atmosphere for $30 \mathrm{~min}$. Phenylacetylene $\left(0.11 \mathrm{~cm}^{3}\right.$, $1 \mathrm{mmol}), 53 \mathrm{mg} \mathrm{Pd} / \mathrm{C}(10 \%, 0.05 \mathrm{mmol})$, and $276 \mathrm{mg}$ $\mathrm{K}_{2} \mathrm{CO}_{3}(2.0 \mathrm{mmol})$ were added, and the resulting solution was heated at $70{ }^{\circ} \mathrm{C}$ for $12 \mathrm{~h}$, and then cooled to room temperature. The reaction mixture was filtered, and the filtrate was concentrated in vacuo. The residue was purified by chromatography, eluting with $\mathrm{CHCl}_{3} / \mathrm{CH}_{3} \mathrm{OH}$ $(98: 2)$ to afford the pure product (Table 2).
1-Phenyl-4-(2-phenylethynyl)[1,2,4]triazolo[4,3-a]quinoxaline $\left(4 \mathbf{a}, \mathrm{C}_{23} \mathrm{H}_{14} \mathrm{~N}_{4}\right)$

White solid; yield $283 \mathrm{mg}$ (82 \%); m.p.: $280-282{ }^{\circ} \mathrm{C}$; $R_{f}=0.53 ;$ IR $(\mathrm{KBr}): \bar{v}=2,200,1,650 \mathrm{~cm}^{-1} ;{ }^{1} \mathrm{H} \mathrm{NMR}$ (400 MHz, DMSO- $\left.d_{6}\right): \delta=7.32-7.42(\mathrm{~m}, 4 \mathrm{H}), 7.45-7.55$ $(\mathrm{m}, 4 \mathrm{H}), 7.67(\mathrm{~d}, J=4.8 \mathrm{~Hz}, 1 \mathrm{H}), 7.81(\mathrm{~d}, J=4.1 \mathrm{~Hz}$, 1H), 7.86-7.92 (m, 3H), 8.05-8.08 (m, 1H) ppm; ${ }^{13} \mathrm{C} \mathrm{NMR}$ (100 MHz, DMSO- $\left.d_{6}\right): \delta=85.26,96.37,123.80,127.55$, $127.85,128.11,128.21,128.70,128.95,129.22,129.55$, $129.76,130.45,132.10,132.45,138.92,142.17,145.11$, $149.12 \mathrm{ppm}$; MS (EI, $70 \mathrm{eV}): m / z(\%)=346\left(\left[\mathrm{M}^{+}\right], 100\right)$, 269 (52), 245 (22), 167 (16); HRMS (ESI): $m / z=$ 346.1201 .

\section{1-(2-Nitrophenyl)-4-(2-phenylethynyl)[1,2,4]triazolo-}

[4,3-a]quinoxaline $\left(\mathbf{4 b}, \mathrm{C}_{23} \mathrm{H}_{13} \mathrm{~N}_{5} \mathrm{O}_{2}\right)$

White solid; yield $367 \mathrm{mg}$ (94\%); m.p.: $284-286{ }^{\circ} \mathrm{C}$; $R_{f}=0.55 ; \mathrm{IR}(\mathrm{KBr}): \bar{v}=2,200,1,600,1,540,1,340 \mathrm{~cm}^{-1}$;

${ }^{1} \mathrm{H}$ NMR (400 MHz, DMSO- $\left.d_{6}\right): \delta=7.56-7.63(\mathrm{~m}, 3 \mathrm{H})$, 7.73-7.75 (m, 1H), 7.80-7.87 (m, 3H), $7.95(\mathrm{dd}, J=6.4$, 
Table 2 Synthesis of triazolo[4,3-a]quinoxalines $\mathbf{4 a}-\mathbf{4} \mathbf{k}^{\mathrm{a}}$

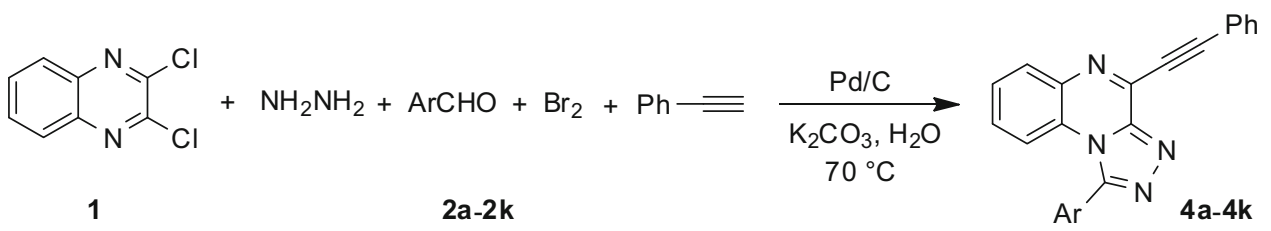

\begin{tabular}{llll}
\hline Entry & $\mathrm{Ar}$ & Product & Yield/\% $^{\mathbf{b}}$ \\
\hline 1 & $\mathrm{C}_{6} \mathrm{H}_{5}$ & $\mathbf{4 a}$ & 82 \\
2 & $2-\mathrm{NO}_{2}-\mathrm{C}_{6} \mathrm{H}_{4}$ & $\mathbf{4 b}$ & 94 \\
3 & $3-\mathrm{NO}_{2}-\mathrm{C}_{6} \mathrm{H}_{4}$ & $\mathbf{4 c}$ & 74 \\
4 & $4-\mathrm{NO}_{2}-\mathrm{C}_{6} \mathrm{H}_{4}$ & $\mathbf{4 d}$ & 94 \\
5 & $2-\mathrm{F}_{6}-\mathrm{C}_{6} \mathrm{H}_{4}$ & $\mathbf{4 e}$ & 76 \\
6 & $4-\mathrm{F}-\mathrm{C}_{6} \mathrm{H}_{4}$ & $\mathbf{4 f}$ & 85 \\
7 & $2-\mathrm{Cl}_{-}-\mathrm{C}_{6} \mathrm{H}_{4}$ & $\mathbf{4 g}$ & 83 \\
8 & $4-\mathrm{Cl}_{-}-\mathrm{C}_{6} \mathrm{H}_{4}$ & $\mathbf{4 h}$ & \\
9 & $2,6-\mathrm{Cl}_{2}-\mathrm{C}_{6} \mathrm{H}_{3}$ & $\mathbf{4 i}$ & \\
10 & $4-\mathrm{CH}_{3}-\mathrm{C}_{6} \mathrm{H}_{4}$ & $\mathbf{4 j}$ & 80 \\
11 & $4-\mathrm{CH}_{3} \mathrm{O}-\mathrm{C}_{6} \mathrm{H}_{4}$ & $\mathbf{4 k}$ \\
\hline
\end{tabular}

${ }^{a}$ Reaction conditions: 1 (1.0 mmol), hydrazine hydrate $(2.0 \mathrm{mmol}), \mathbf{2 a}-\mathbf{2 k}(1.0 \mathrm{mmol})$, phenylacetylene $(1.0 \mathrm{mmol})$, bromine $(1.0 \mathrm{mmol})$, $\mathrm{K}_{2} \mathrm{CO}_{3}(2.0 \mathrm{mmol}), 10 \% \mathrm{Pd} / \mathrm{C}(5 \mathrm{~mol} \%), 5 \mathrm{~cm}^{3}$ degassed water, $70{ }^{\circ} \mathrm{C}, 10 \mathrm{~h}$

b Isolated yields

Scheme 2

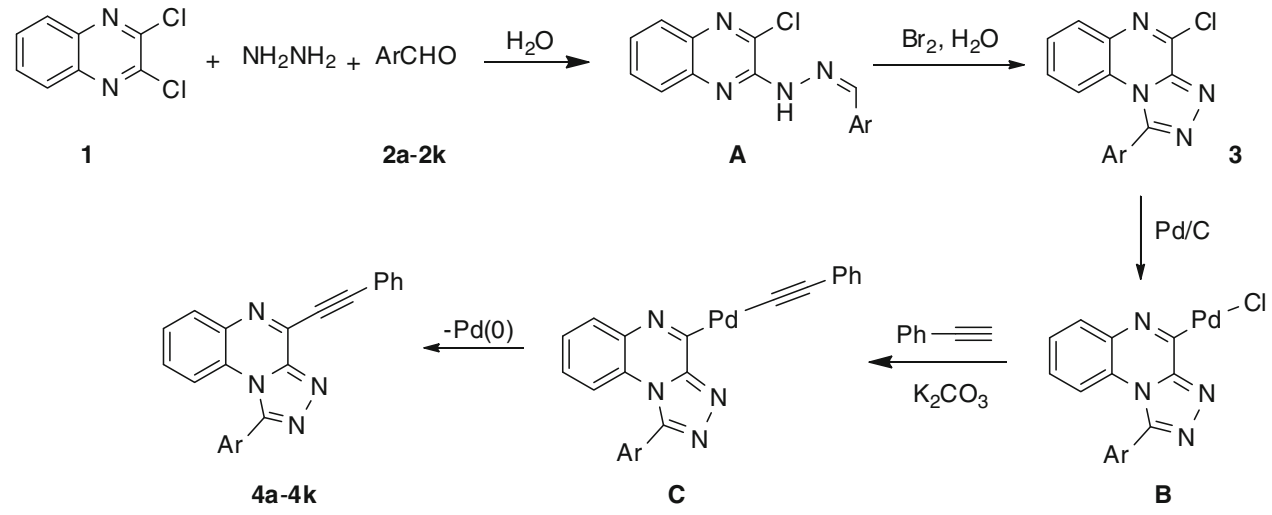

$2.0 \mathrm{~Hz}, 2 \mathrm{H}), 8.05-8.10(\mathrm{~m}, 2 \mathrm{H}), 8.16-8.19(\mathrm{~m}, 2 \mathrm{H}) \mathrm{ppm}$;

${ }^{13} \mathrm{C}$ NMR $\left(100 \mathrm{MHz}, \mathrm{DMSO}-d_{6}\right): \delta=85.39,96.45$, $121.36,122.72,127.45,128.25,128.35,128.47,128.66$, $129.42,129.58,129.68,131.36,132.45,132.60,135.54$, 142.36, 145.78, $149.25 \mathrm{ppm} ; \mathrm{MS}$ (EI, $70 \mathrm{eV}): \mathrm{m} / \mathrm{z}$ $(\%)=391\left(\left[\mathrm{M}^{+}\right], 100\right), 345$ (32), 314 (51), 290 (21), 169 (24); HRMS (ESI): $m / z=391.1037$.

1-(3-Nitrophenyl)-4-(2-phenylethynyl)[1,2,4]triazolo[4,3-a]quinoxaline $\left(4 \mathrm{c}, \mathrm{C}_{23} \mathrm{H}_{13} \mathrm{~N}_{5} \mathrm{O}_{2}\right)$

White solid; yield $289 \mathrm{mg}$ (74 \%); m.p.: $274-276{ }^{\circ} \mathrm{C}$; $R_{f}=0.52 ;$ IR $(\mathrm{KBr}): \bar{v}=2,230,1,610,1,530,1,340 \mathrm{~cm}^{-1}$; ${ }^{1} \mathrm{H} \mathrm{NMR}\left(400 \mathrm{MHz}, \mathrm{DMSO}-d_{6}\right): \delta=7.28(\mathrm{~s}, 1 \mathrm{H}), 7.35-7.40$ (m, 2H), 7.46-7.52 (m, 3H), 7.54-7.60 (m, 3H), 7.64-7.71 (m,
3H), 8.03-8.06 (m, 1H) ppm; ${ }^{1} \mathrm{C}$ NMR (100 MHz, DMSO$\left.d_{6}\right): \delta=85.26,96.35,121.10,122.45,127.52,128.45$, $129.26,129.45,129.64,129.88,130.20,131.36,132.25$, 132.45, 142.36, 145.56, 148.92, 149.55 ppm; MS (EI, $70 \mathrm{eV})$ : $m / z(\%)=391\left(\left[\mathrm{M}^{+}\right], 100\right), 345$ (35), 314 (43), 290 (26), 167 (21); HRMS (ESI): $m / z=391.1042$.

1-(4-Nitrophenyl)-4-(2-phenylethynyl)[1,2,4]triazolo[4,3-a]quinoxaline $\left(\mathbf{4 d}, \mathrm{C}_{23} \mathrm{H}_{13} \mathrm{~N}_{5} \mathrm{O}_{2}\right)$

White solid; yield $367 \mathrm{mg}$ (94\%); m.p.: $293-295{ }^{\circ} \mathrm{C}$; $R_{f}=0.54 ;$ IR $(\mathrm{KBr}): \bar{v}=2,210,1,620,1,540,1,320 \mathrm{~cm}^{-1}$; ${ }^{1} \mathrm{H}$ NMR (400 MHz, DMSO- $\left.d_{6}\right): \delta=7.40(\mathrm{~d}, J=8.4 \mathrm{~Hz}$, 1H), 7.55-7.61 (m, 4H), 7.68-7.71 (m, 1H), 7.80-7.86 (m, $2 \mathrm{H}), 8.11-8.18(\mathrm{~m}, 3 \mathrm{H}), 8.55(\mathrm{~d}, J=8.2 \mathrm{~Hz}, 2 \mathrm{H}) \mathrm{ppm} ;{ }^{13} \mathrm{C}$ 
NMR (100 MHz, DMSO- $\left.d_{6}\right): \delta=84.95,96.35,116.61$, $120.60,124.75,126.02,128.50,129.68,130.72,131.29$, $132.14,132.85,134.87,135.97,136.09,145.33,148.53$, $149.46 \mathrm{ppm}$; MS (EI, $70 \mathrm{eV}): \mathrm{m} / z(\%)=391\left(\left[\mathrm{M}^{+}\right], 100\right)$, 345 (40), 314 (47), 290 (18), 168 (29); HRMS (ESI): $\mathrm{m} / \mathrm{z}=391.1047$.

\section{1-(2-Fluorophenyl)-4-(2-phenylethynyl) [1,2,4]triazolo-}

[4,3-a]quinoxaline $\left(4 \mathbf{e}, \mathrm{C}_{23} \mathrm{H}_{13} \mathrm{FN}_{4}\right)$

White solid; yield $276 \mathrm{mg}$ (76\%); m.p.: $305-307{ }^{\circ} \mathrm{C}$; $R_{f}=0.50 ; \mathrm{IR}(\mathrm{KBr}): \bar{v}=2,250,1,570 \mathrm{~cm}^{-1} ;{ }^{1} \mathrm{H} \mathrm{NMR}$ $\left(400 \mathrm{MHz}, \mathrm{DMSO}-d_{6}\right): \delta=7.20(\mathrm{~s}, 1 \mathrm{H}), 7.35-7.43(\mathrm{~m}$, $4 \mathrm{H}), 7.57-7.64(\mathrm{~m}, 4 \mathrm{H}), 7.82-7.86(\mathrm{~m}, 2 \mathrm{H}), 8.21(\mathrm{~d}$, $J=8.0 \mathrm{~Hz}, 2 \mathrm{H}) \mathrm{ppm} ;{ }^{13} \mathrm{C}$ NMR $\left(100 \mathrm{MHz}\right.$, DMSO- $\left.d_{6}\right)$ : $\delta=85.80,96.42,116.62,122.26,122.92,124.85,127.70$, $128.37,128.81,129.21,130.35,130.56,133.51,133.65$, $141.52,146.23,146.56,149.55,160.42 \mathrm{ppm}$; MS (EI, $70 \mathrm{eV}): \mathrm{m} / z(\%)=364\left(\left[\mathrm{M}^{+}\right], 100\right), 345(27), 287(62)$, 263 (42), 167 (25); HRMS (ESI): $m / z=364.1152$.

1-(4-Fluorophenyl)-4-(2-phenylethynyl)[1,2,4]triazolo[4,3-a]quinoxaline $\left(\mathbf{4 f}, \mathrm{C}_{23} \mathrm{H}_{13} \mathrm{FN}_{4}\right)$

White solid; yield $309 \mathrm{mg}$ (85\%); m.p.: $313-315{ }^{\circ} \mathrm{C}$; $R_{f}=0.51 ;$ IR $(\mathrm{KBr}): \bar{v}=2,230,1,590 \mathrm{~cm}^{-1} ;{ }^{1} \mathrm{H}$ NMR $\left(400 \mathrm{MHz}\right.$, DMSO- $\left.d_{6}\right): \delta=7.20(\mathrm{~s}, 1 \mathrm{H}), 7.35-7.41(\mathrm{~m}$, $2 \mathrm{H}), 7.55-7.62(\mathrm{~m}, 2 \mathrm{H}), 7.78-7.82(\mathrm{~m}, 2 \mathrm{H}), 7.90-7.98(\mathrm{~m}$, $4 \mathrm{H}), 8.15-8.19(\mathrm{~m}, 2 \mathrm{H}) \mathrm{ppm} ;{ }^{13} \mathrm{C}$ NMR (100 MHz, DMSO- $\left.d_{6}\right): \delta=85.82,96.44,116.36,116.45,122.32$, $126.22,127.32,128.24,128.55,128.74,128.92,129.20$, $130.35,130.47,132.10,132.21,142.30,145.25,149.25$, $162.92 \mathrm{ppm} ; \mathrm{MS}(\mathrm{EI}, 70 \mathrm{eV}): \mathrm{m} / \mathrm{z}(\%)=364\left(\left[\mathrm{M}^{+}\right], 100\right)$, 345 (32), 287 (67), 263 (35), 167 (29); HRMS (ESI): $m / z=364.1144$.

1-(2-Chlorophenyl)-4-(2-phenylethynyl) [1,2,4]triazolo-

[4,3-a]quinoxaline $\left(\mathbf{4 g}, \mathrm{C}_{23} \mathrm{H}_{13} \mathrm{ClN}_{4}\right)$

White solid; yield $315 \mathrm{mg}$ (83\%); m.p.: $276-278{ }^{\circ} \mathrm{C}$; $R_{f}=0.56 ;$ IR $(\mathrm{KBr}): \bar{v}=2,250,1,600 \mathrm{~cm}^{-1} ;{ }^{1} \mathrm{H}$ NMR $\left(400 \mathrm{MHz}\right.$, DMSO- $\left.d_{6}\right): \delta=7.35(\mathrm{~s}, 1 \mathrm{H}), 7.43-7.52(\mathrm{~m}$, $4 \mathrm{H})$, 7.60-7.70 (m, 4H), 8.06-8.12 (m, 2H), $8.40(\mathrm{~d}$, $J=8.0 \mathrm{~Hz}, 2 \mathrm{H}) \mathrm{ppm} ;{ }^{13} \mathrm{C}$ NMR $\left(100 \mathrm{MHz}\right.$, DMSO- $\left.d_{6}\right)$ : $\delta=85.65,96.53,123.42,127.53,127.65,128.21,128.32$, $128.42,128.65,129.25,129.64,130.36,130.54,132.25$, 132.36, 132.46, 138.65, 142.35, 145.65, 149.58 ppm; MS $($ EI, $\quad 70 \mathrm{eV}): \quad m / z \quad(\%)=382 \quad\left(\left[\mathrm{M}^{+}\right]\left[{ }^{37} \mathrm{Cl}\right], \quad 31\right), \quad 380$ $\left(\left[\mathrm{M}^{+}\right]\left[{ }^{35} \mathrm{Cl}\right], 100\right), 345$ (35), 303 (57), 279 (21), 167 (25); HRMS (ESI): $m / z=380.0850$.

1-(4-Chlorophenyl)-4-(2-phenylethynyl) [1,2,4]triazolo[4,3-a]quinoxaline (4h, $\left.\mathrm{C}_{23} \mathrm{H}_{13} \mathrm{ClN}_{4}\right)$

White solid; yield $326 \mathrm{mg}$ (86\%); m.p.: $289-291{ }^{\circ} \mathrm{C}$; $R_{f}=0.55 ;$ IR $(\mathrm{KBr}): \bar{v}=2,240,1,600 \mathrm{~cm}^{-1} ;{ }^{1} \mathrm{H}$ NMR $\left(400 \mathrm{MHz}, \mathrm{DMSO}-d_{6}\right): \delta=7.41-7.45(\mathrm{~m}, 1 \mathrm{H}), 7.55-7.63$ $(\mathrm{m}, 4 \mathrm{H}), 7.65-7.69(\mathrm{~m}, 1 \mathrm{H}), 7.71-7.77(\mathrm{~m}, 3 \mathrm{H}), 7.85(\mathrm{~d}$, $J=8.0 \mathrm{~Hz}, 2 \mathrm{H}), 8.11-8.18(\mathrm{~m}, 2 \mathrm{H}) \mathrm{ppm} ;{ }^{13} \mathrm{C} \mathrm{NMR}$ $\left(100 \mathrm{MHz}, \mathrm{DMSO}-d_{6}\right): \delta=85.01,96.20,116.25,120.63$, $126.18,127.49,128.34,129.68,129.85,130.60,131.27$, $132.45,132.83,136.03,136.46,136.80,145.11,149.14$ ppm; MS (EI, $70 \mathrm{eV}): m / z(\%)=382\left(\left[\mathrm{M}^{+}\right]\left[{ }^{37} \mathrm{Cl}\right], 32\right), 380$ ([ $\left.\left.\mathrm{M}^{+}\right]\left[{ }^{35} \mathrm{Cl}\right], 100\right), 345$ (30), 303 (62), 279 (26), 168 (20); HRMS (ESI): $m / z=380.0850$.

1-(2,6-Dichlorophenyl)-4-(2-phenylethynyl) [1,2,4]triazolo[4,3-a]quinoxaline $\left(4 \mathbf{i}, \mathrm{C}_{23} \mathrm{H}_{12} \mathrm{Cl}_{2} \mathrm{~N}_{4}\right)$

White solid; yield $310 \mathrm{mg}$ (75\%); m.p.: $267-269^{\circ} \mathrm{C}$; $R_{f}=0.57 ; \mathrm{IR}(\mathrm{KBr}): \bar{v}=2,210,1,620 \mathrm{~cm}^{-1} ;{ }^{1} \mathrm{H} \mathrm{NMR}$ $\left(400 \mathrm{MHz}, \mathrm{DMSO}-d_{6}\right): \delta=7.20(\mathrm{~s}, 1 \mathrm{H}), 7.40-7.48(\mathrm{~m}$, $4 \mathrm{H}), 7.55-7.62(\mathrm{~m}, 3 \mathrm{H}), 7.75-7.78(\mathrm{~m}, 2 \mathrm{H}), 8.12-8.15(\mathrm{~m}$, $1 \mathrm{H}), 8.17-8.20(\mathrm{~m}, 1 \mathrm{H}) \mathrm{ppm} ;{ }^{13} \mathrm{C}$ NMR $(100 \mathrm{MHz}$, DMSO- $\left.d_{6}\right): \delta=85.35,96.23,127.80,127.95,128.44$, $128.84,128.92,129.10,129.25,129.54,130.92,131.44$, $132.35,132.74,133.55,138.22,142.62,145.84,149.12 \mathrm{ppm}$; MS (EI, $70 \mathrm{eV}): \mathrm{m} / \mathrm{z}(\%)=418\left(\left[\mathrm{M}^{+}\right]\left[{ }^{37} \mathrm{Cl}_{2}\right], 12\right), 416$ $\left(\left[\mathrm{M}^{+}\right]\left[{ }^{37} \mathrm{Cl}\right]\left[{ }^{35} \mathrm{Cl}\right], 53\right), 414\left(\left[\mathrm{M}^{+}\right]\left[{ }^{35} \mathrm{Cl}_{2}\right], 100\right), 381(23)$, 339 (65), 313 (30), 269 (32), 168 (15); HRMS (ESI): $\mathrm{m} / \mathrm{z}=414.0475$.

\section{1-(4-Methylphenyl)-4-(2-phenylethynyl) [1,2,4]triazolo-}

[4,3-a]quinoxaline $\left(\mathbf{4} \mathbf{j}, \mathrm{C}_{24} \mathrm{H}_{16} \mathrm{~N}_{4}\right)$

White solid; yield $288 \mathrm{mg}(80 \%)$; m.p.: $218-220{ }^{\circ} \mathrm{C}$; $R_{f}=0.52 ; \mathrm{IR}(\mathrm{KBr}): \bar{v}=2,220,1,600 \mathrm{~cm}^{-1} ;{ }^{1} \mathrm{H}$ NMR $\left(400 \mathrm{MHz}, \quad\right.$ DMSO- $\left.d_{6}\right): \delta=2.39 \quad(\mathrm{~s}, \quad 3 \mathrm{H}), 7.36 \quad(\mathrm{~d}$, $J=8.0 \mathrm{~Hz}, 2 \mathrm{H}), 7.50-7.62(\mathrm{~m}, 4 \mathrm{H}), 7.70-7.77(\mathrm{~m}, 3 \mathrm{H})$, 8.08-8.14 (m, 2H), 8.40-8.46 (m, 2H) ppm; ${ }^{13} \mathrm{C}$ NMR $\left(100 \mathrm{MHz}, \mathrm{DMSO}-d_{6}\right): \delta=22.62,85.12,96.21,116.25$, $120.53,124.62,126.10,128.42,128.60,129.52,129.92$, $130.62,132.34,132.45,138.40,140.92,141.52,145.22$, $149.62 \mathrm{ppm}$; MS (EI, $70 \mathrm{eV}): \mathrm{m} / \mathrm{z}(\%)=360\left(\left[\mathrm{M}^{+}\right], 100\right)$, 345 (21), 283 (64), 259 (24), 168 (22); HRMS (ESI): $m / z=360.1343$.

1-(4-Methoxyphenyl)-4-(2-phenylethynyl) [1,2,4]triazolo[4,3-a]quinoxaline $\left(\mathbf{4} \mathbf{k}, \mathrm{C}_{24} \mathrm{H}_{16} \mathrm{~N}_{4} \mathrm{O}\right)$

White solid; yield $304 \mathrm{mg}$ (81\%); m.p.: $250-252{ }^{\circ} \mathrm{C}$; $R_{f}=0.51 ; \mathrm{IR}(\mathrm{KBr}): \bar{v}=2,200,1,620 \mathrm{~cm}^{-1} ;{ }^{1} \mathrm{H}$ NMR $\left(400 \mathrm{MHz}, \quad\right.$ DMSO- $\left.d_{6}\right): \quad \delta=3.90 \quad(\mathrm{~s}, \quad 3 \mathrm{H}), 7.20 \quad(\mathrm{~d}$, $J=8.7 \mathrm{~Hz}, 2 \mathrm{H}), 7.41-7.52(\mathrm{~m}, 3 \mathrm{H}), 7.54-7.62(\mathrm{~m}, 3 \mathrm{H})$, $7.67-7.70 \quad(\mathrm{~m}, \quad 1 \mathrm{H}), \quad 7.75-7.81 \quad(\mathrm{~m}, \quad 2 \mathrm{H}), 8.06 \quad(\mathrm{~d}$, $J=8.4 \mathrm{~Hz}, 2 \mathrm{H}) \mathrm{ppm} ;{ }^{13} \mathrm{C}$ NMR $\left(100 \mathrm{MHz}\right.$, DMSO- $\left.d_{6}\right)$ : $\delta=55.92,85.15,96.20,116.12,122.36,124.51,126.15$, $128.21,128.42,128.52,128.95,129.55,129.78,130.54$, $132.32,132.45,140.48,145.23,149.65 \mathrm{ppm}$; MS (EI, $70 \mathrm{eV}): m / z(\%)=376\left(\left[\mathrm{M}^{+}\right], 100\right), 345(21), 299(64)$, 275 (27), 269 (32), 168 (27); HRMS (ESI): $m / z=$ 376.1360 .

Acknowledgments We are grateful to the Research Council of Shahrood University of Technology for the financial support of this work. 
Open Access This article is distributed under the terms of the Creative Commons Attribution License which permits any use, distribution, and reproduction in any medium, provided the original author(s) and the source are credited.

\section{References}

1. Hazeldine ST, Polin L, Kushner J, Paluch J, White K, Edelstein M, Palomino E, Corbett TH, Horwitz JP (2001) J Med Chem 44:1758

2. Rong F, Chow S, Yan S, Larson G, Hong Z, Wu J (2007) Bioorg Med Chem Lett 17:1663

3. Jaso A, Zarranz B, Aldana I, Monge A (2005) J Med Chem 48:2019

4. Smits RA, Lim HD, Hanzer A, Zuiderveld OP, Guaita E, Adami M, Coruzzi G, Leurs R, de Esch IJP (2008) J Med Chem 51:2457

5. Cheeseman GWH, Werstiuk ESG (1978) Adv Heterocycl Chem 22:367

6. Colotta V, Catarzi D, Varano F, Lenzi O, Filacchioni G, Martini C, Trincavelli L, Ciampi O, Traini C, Pugliese AM, Pedata F, Morizzo E, Moro S (2008) Bioorg Med Chem 16:6086

7. Sonogashira K, Tohda Y, Hagihara N (1975) Tetrahedron Lett $16: 4467$

8. Chinchilla R, Nájera C (2007) Chem Rev 107:874

9. Chinchilla R, Nájera C (2011) Chem Soc Rev 40:5084

10. Paterson I, Davies RDM, Marquez R (2001) Angew Chem Int Ed 40:603

11. Cosford NDP, Tehrani L, Roppe J, Schweiger E, Smith ND, Anderson J, Bristow L, Brodkin J, Jiang X, McDonald I, Rao S, Washburn M, Varney M (2003) J Med Chem 46:204

12. Mongin O, Porres L, Moreaux L, Mertz J, Blanchard-Desce M (2002) Org Lett 4:719

13. Onitsuka K, Fujimoto M, Ohshiro N, Takahashi S (1999) Angew Chem Int Ed 38:689

14. Raju S, Kumar PR, Mukkanti K, Annamalai P, Pal M (2006) Bioorg Med Chem Lett 16:6185
15. Reddy EA, Barange DK, Islam A, Mukkanti K, Pal M (2008) Tetrahedron 64:7143

16. Batchu VR, Subramanian V, Parasuraman K, Swamy NK, Kumar S, Pal M (2005) Tetrahedron 61:9869

17. Komáromi A, Tolnai GL, Novák Z (2008) Tetrahedron Lett 49:7294

18. Ruiz J, Cutillas N, Lopez F, Lopez G, Bautista D (2006) Organometallics 25:5768

19. Consorti CS, Flores FR, Rominger F, Dupont J (2006) Adv Synth Catal 348:133

20. Dhudshia B, Thadani AN (2006) Chem Commun 46:668

21. Yi CY, Hua RM (2006) Catal Commun 7:377

22. Komáromi A, Novák Z (2008) Chem Commun 40:4968

23. Yi CY, Hua RM (2006) J Org Chem 71:2535

24. Soheili A, Albaneze-Walker J, Murry JA, Dormer PG, Hughes DL (2003) Org Lett 5:4191

25. Herrmann WA (2002) Angew Chem Int Ed 41:1290

26. Gholap AR, Venkatesan K, Pasricha R, Daniel T, Lahoti RJ, Srinivasan KV (2005) J Org Chem 70:4869

27. Fleckenstein CA, Plenio H (2008) Green Chem 10:563

28. Glaser C (1869) Ber Dtsch Chem Ges 2:422

29. Hay AS (1962) J Org Chem 27:3320

30. Siemsen P, Livingston RC, Diederich F (2000) Angew Chem Int Ed 39:2632

31. Mironov MA (2006) QSAR Comb Sci 25:423

32. Dömling A (2006) Chem Rev 106:17

33. Ramón DJ, Yus M (2005) Angew Chem Int Ed 44:1602

34. DeVasher RB, Moore LR, Shaughnessy KH (2004) J Org Chem 69:7919

35. Anderson KW, Buchwald SL (2005) Angew Chem Int Ed 44:6173

36. Mio MJ, Kopel LC, Braun JB, Gadzikwa TL, Hull KL, Brisbois RG, Markworth CJ, Grieco PA (2002) Org Lett 4:3199

37. Bhattacharya S, Sengupta S (2004) Tetrahedron Lett 45:8733

38. Bakherad M, Keivanloo A, Jajarmi S (2012) Tetrahedron 68:2107

39. Keivanloo A, Bakherad M, Rahimi A, Taheri SAN (2010) Tetrahedron Lett 51:2409

40. Keivanloo A, Bakherad M, Rahimi A (2010) Synthesis 42:1599

41. Cheeseman GWH (1962) J Chem Soc 1:1170 